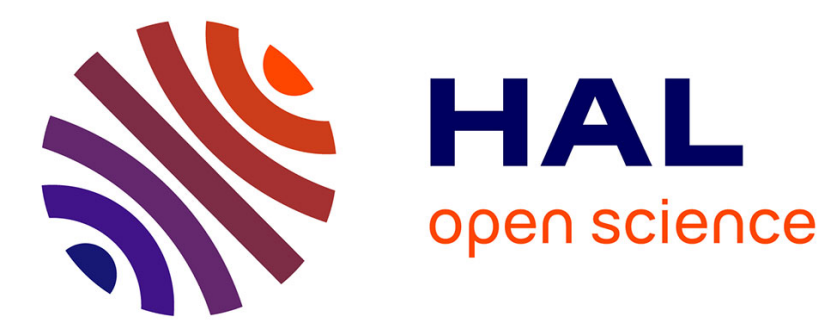

\title{
Zero temperature magnetization of a one-dimensional spin glass
}

\author{
E. Gardner, Bernard Derrida
}

\section{To cite this version:}

E. Gardner, Bernard Derrida. Zero temperature magnetization of a one-dimensional spin glass. Journal of Statistical Physics, 1985, 39 (3-4), pp.367-377. 10.1007/BF01018668 . hal-03285918

\section{HAL Id: hal-03285918 \\ https://hal.science/hal-03285918}

Submitted on 21 Jul 2021

HAL is a multi-disciplinary open access archive for the deposit and dissemination of scientific research documents, whether they are published or not. The documents may come from teaching and research institutions in France or abroad, or from public or private research centers.
L'archive ouverte pluridisciplinaire HAL, est destinée au dépôt et à la diffusion de documents scientifiques de niveau recherche, publiés ou non, émanant des établissements d'enseignement et de recherche français ou étrangers, des laboratoires publics ou privés. 


\title{
Zero Temperature Magnetization of a One-Dimensional Spin Glass
}

\author{
E. Gardner ${ }^{1}$ and B. Derrida ${ }^{2}$
}

Received October 12, 1984

\begin{abstract}
We calculate the zero temperature magnetization $m$ of a one-dimensional Ising spin glass is a weak magnetic field $h$. We show that $m \sim C h^{x}$ and give closed expressions for the constant $C$ and the exponent $x$ which both depend on the probability distribution of nearest-neighbor interactions.
\end{abstract}

KEY WORDS: Spin glass; Ising chain; magnetization.

\section{INTRODUCTION}

The existence of a spin glass phase in the real world (three dimensions) has been for many years a very controversial subject and it is not yet established whether three-dimensional spin glasses exhibit a phase transition at zero or at finite temperature.

In the case of one-dimensional spin glasses (and probably also in two dimensions), the situation is much simpler because the phase transition occurs at zero temperature. It is then interesting to know the critical behavior which characterizes this zero temperature transition. In two dimensions, the problem seems too difficult to avoid numerical methods. ${ }^{(1,3)}$ On the other hand, in one dimension the problem is simple enough to allow analytic calculations at zero temperature.

The Hamiltonian of a one-dimensional Ising spin glass can be written as

$$
\mathscr{H}=-\sum_{i} J_{i, i+1} \sigma_{i} \sigma_{i+1}-h \sum_{i} \sigma_{i}
$$

\footnotetext{
${ }^{1}$ Service de Physique Théorique, CEN-Saclay, 91191 Gif sur Yvette, Cedex, France.

${ }^{2}$ Service de Physique Théorique, CEN-Saclay, 91191 Gif sur Yvette, Cedex, France and Department of Physics, The Weizmann Institute of Science, Israel.
} 
where the $\sigma_{i}$ are Ising spins $\left(\sigma_{i}= \pm 1\right)$ and the interactions $J_{i, i+1}$ are independent random variables distributed according to a given probability distribution $\rho\left(J_{i, i+1}\right)$.

Several authors have already considered the problem of calculating the zero temperature properties of such a chain for special distribution $\rho^{(4-7)}$ : in particular when $\rho\left(J_{i j}\right)$ is a mixture of ferromagnetic and antiferromagnetic bonds of equal strengths $\left[\rho\left(J_{i j}\right)=(1-x) \delta\left(J_{i j}-J\right)+x \delta\left(J_{i j}+J\right)\right]$, the ground state energy, entropy, and magnetization can be calculated exactly $^{(4-6)}$ for any value of the magnetic field $h$. One can also obtain exact results for other one-dimensional disordered models like ferromagnetic Ising chains in a random magnetic field ${ }^{(8)}$ but only for special distributions of the random field. ${ }^{(6,9,10)}$ However much less is known for general distributions $\rho\left(J_{i j}\right)$ of interactions or for general distributions of the random field. ${ }^{(11,12)}$

Recently Chen and $\mathrm{Ma}^{(13)}$ attacked the problem of a general distribution $\rho\left(J_{i j}\right)$ and they showed that the zero temperature magnetization is nonanalytic at $h=0$ and has the following behavior:

$$
m \simeq C h^{(K+1) /(K+3)} \quad \text { for } h \rightarrow 0
$$

where the parameter $K$ which appears in the exponent is given by the shape of the probability distribution $\rho\left(J_{i j}\right)$ at $J_{i j}=0$ :

$$
\rho\left(J_{i j}\right) \sim A\left|J_{i j}\right|^{K}
$$

Their argument is beautiful and rather simple: in a weak magnetic field, the magnetization is due to the flip of clusters which are delimited by two weak bonds: the typical distance $D$ between two bonds weaker than $\left|J_{0}\right|$ behaves like

$$
D=\left[\int_{-\left|J_{0}\right|}^{\left|J_{0}\right|} \rho\left(J_{i j}\right) d J_{i j}\right]^{-1} \sim\left|J_{0}\right|^{-(K+1)}
$$

The typical magnetization at $T=0$ of a cluster of length $D$ is $\sqrt{D} \sim$ $\left|J_{0}\right|^{-(K+1) / 2}$. Therefore the typical cluster of length $D$ delimited by two weak bonds flips for a value of the magnetic field $h \sim\left|J_{0}\right| / \sqrt{D}$ i.e., $h \sim\left|J_{0}\right|^{(K+3) / 2}$. The typical magnetization per spin of the chain due to the flip of these clusters is therefore $m \simeq D^{1 / 2} / D \sim\left|J_{0}\right|^{(K+1) / 2}$ i.e., $m \sim H^{(K+1) /(K+3)}$. Although this argument gives a simple and physical reason for the nonanalytic behavior of $m$ at $h=0$, it is not easy to take into account the fact that there may be flipped clusters inside flipped clusters and it does not allow one to calculate the constant $C$ which appears in Eq. (2).

The purpose of the present paper is to derive formula (2) for an arbitrary distribution which behaves as in Eq. (3) for $J \rightarrow 0$ and to find the 
expression for the constant $C$ in terms of the distribution $\rho$. Our final result will be that for small $h$ :

$$
\begin{aligned}
m \simeq & h^{(K+1) /(K+3)}\left(\frac{\alpha}{1-\alpha}\right)^{(K+2) /(K+3)}\left[\frac{4 A}{(K+1)(K+3)^{2}}\right]^{1 /(K+3)} \\
& \times(K+2) \frac{\Gamma((K+2) /(K+3))}{\Gamma(1 /(K+3))}
\end{aligned}
$$

where the parameters $A$ and $K$ are the ones which appear in (3), and the constant $\alpha$ is defined by

$$
\int_{0}^{\infty} \rho\left(J_{i j}\right) d J_{i j}=\alpha
$$

So $\alpha$ is the density of ferromagnetic bonds. The technique used to obtain this result is based on transfer matrix ideas.

\section{A FORMULATION VALID FOR ANY FIELD $h$}

The idea of our calculation is very similar to the one which was used for the $\pm J$ case. $^{(3)}$ One writes a recursion relation for the ground state energy. The Hamiltonian $\mathscr{H}_{L+1}$ of a chain of $L+1$ spins can be decomposed into the Hamiltonian $\mathscr{H}_{L}$ of a chain of $L$ spins plus the part of $\mathscr{H}_{L+1}$ which contains the spin $\sigma_{L+1}$

$$
\mathscr{H}_{L+1}=\mathscr{H}_{L}-J_{L, L+1} \sigma_{L} \sigma_{L+1}-h \sigma_{L+1}
$$

Let us denote by $-F_{L}$ the ground state energy of the chain of $L$ spins if the spin $\sigma_{L}=+1$ and $-G_{L}$ this ground state energy if $\sigma_{L}=-1$. Clearly one has

$$
\begin{aligned}
& F_{L+1}=h+\max \left(F_{L}+J_{L, L+1} ; G_{L}-J_{L, L+1}\right) \\
& G_{L+1}=-h+\max \left(F_{L}-J_{L, L+1} ; G_{L}+J_{L, L+1}\right)
\end{aligned}
$$

These recursion relations express the fact that to find the ground state energy of $\mathscr{H}_{L+1}$, one has to find the value of $\sigma_{L}$ which minimizes $\mathscr{H}_{L+1}$.

$F_{L}$ and $G_{L}$ increase with $L$ but their difference remains finite. If we define $C_{L}$ by

$$
2 C_{L}=G_{L}-F_{L}
$$

then Eqs. (8) and (9) give

$$
C_{L+1}=C_{L}-h+\max \left(-C_{L}-J_{L, L+1} ; 0\right)-\max \left(C_{L}-J_{L, L+1} ; 0\right)
$$


One has always

$$
\left|C_{L+1}\right| \leqslant h+\left|J_{L, L+1}\right|
$$

and so $C_{L}$ does not increase with $L$.

For large $L, C_{L}$ has a stationary probability distribution $P(c)$ which satisfies

$$
\begin{aligned}
P(c)= & \int \rho(J) d J \int P\left(c^{\prime}\right) d c^{\prime} \delta\left(c-c^{\prime}+h-\max \left(-c^{\prime}-J ; 0\right)\right. \\
& \left.+\max \left(c^{\prime}-J ; 0\right)\right)
\end{aligned}
$$

This integral equation can be rewritten as

$$
\begin{aligned}
P(c-h)= & \rho(-c) \int_{-\infty}^{-|c|} P\left(c^{\prime}\right) d c^{\prime}+\rho(c) \int_{|c|}^{\infty} P\left(c^{\prime}\right) d c^{\prime} \\
& +P(c) \int_{|c|}^{\infty} \rho(J) d J+P(-c) \int_{-\infty}^{-|c|} \rho(J) d J
\end{aligned}
$$

It is not difficult to obtain (14) from (13); one way of doing it is to break the integral over $J$ into two parts: one from $-\infty$ to 0 and the other from 0 to $+\infty$. By considering the different cases, one gets (14).

If one is able to find the distribution $P(c)$ which solves the integral equation (14), then the ground state energy $-E$ would be known in the thermodynamic limit:

$$
\begin{aligned}
E & =\lim _{L \rightarrow \infty} \frac{F_{L}}{L}=\lim _{L \rightarrow \infty} \frac{G_{L}}{L}=\lim _{L \rightarrow \infty} \frac{F_{L}+G_{L}}{2 L} \\
& =\frac{1}{2} \lim _{L \rightarrow \infty}\left\langle F_{L+1}+G_{L+1}-F_{L}-G_{L}\right\rangle
\end{aligned}
$$

where in (15) the average means an average over $J_{L, L+1}$ and over $C_{L}$. Since from (8), (9), and (10), we have

$$
\begin{aligned}
F_{L+1}+G_{L+1}-F_{L}-G_{L}= & 2 J_{L, L+1}+\max \left(0 ; 2 C_{L}-2 J_{L, L+1}\right) \\
& +\max \left(-2 C_{L}-2 J_{L, L+1} ; 0\right)
\end{aligned}
$$

Therefore

$$
\begin{aligned}
E= & \int_{-\infty}^{+\infty} J \rho(J) d J+\int_{-\infty}^{+\infty} \rho(J) d J \int_{J}^{\infty} P(c)(c-J) d c \\
& +\int_{-\infty}^{+\infty} \rho(J) d J \int_{-\infty}^{-J} P(c)(-c-J) d c
\end{aligned}
$$


For any distribution $\rho(J)$ and for any value of $h$, the ground state energy is given by (17) is one knows how to solve (14). Then the magnetization $m$ can be obtained by taking the derivative of $E$ with respect to $h$. One knows the solution of (14) only for special distributions like, for example, the $\pm J$ case. ${ }^{(3)} \mathrm{We}$ are going to see in the next section that in the limit $h \ll 1$, one can solve the integral equation (14) for any distribution of the $J$ s.

\section{THE LIMIT OF A WEAK MAGNETIC FIELD}

In a weak magnetic field, the integral equation (14) leads to a differential equation which can be solved.

Without any approximation, one can replace (14) by

$$
\begin{aligned}
P(c-h)-\alpha P(c)-(1-\alpha) P(-c) & \\
= & \rho(c) \int_{|c|}^{\infty} P\left(c^{\prime}\right) d c^{\prime}+\rho(-c) \int_{-\infty}^{-|c|} P\left(c^{\prime}\right) d c^{\prime} \\
& \quad-P(c) \int_{0}^{|c|} \rho(J) d J-P(-c) \int_{-|c|}^{0} \rho(J) d J
\end{aligned}
$$

where $\alpha$ is defined by formula (6).

For $h=0$, the number of solutions of this equation depends on the support of $\rho(J)$. There is always the solution $P(c)=\delta(c)$. But if there is a minimal value $|J|_{\min }$ of $|J|$, i.e., 0 does not belong to the support of $\rho(J)$ any symmetric $[P(c)=P(-c)]$ distribution $P(c)$ whose support is in ]$-|J|_{\min },|J|_{\min }[$ is a solution of (18). If we limit ourselves to the case of distributions which have the behavior (3), then $|J|_{\min }=0$ for $h=0$

$$
P(c)=\delta(c)
$$

We are now going to see that the distribution $P(c)$ which depends on $h$ and $c$ takes in the limit $h \rightarrow 0$ a scaling form

$$
P(c)+P(-c) \sim h^{-2 /(K+3)} \chi\left(h^{-2 /(K+3)} c\right)
$$

To show that (20) is valid in the limit $h \rightarrow 0$, we shall use a self-consistent argument: assuming that (20) is valid, we shall simplify Eq. (18). Then we shall solve the simplified equation and show that it agrees with (20).

The form (20) means that $P(c)$ is concentrated in a region of size $h^{2 /(K+3)}$ around 0 . In this region, one can replace $\rho(c)$ by its asymptotic 
form (3) and one can expand $P(c-h)$ in powers of $h$ since $\chi$ will have a small variation between $c$ and $c-h$. So for $h$ small, one can replace (18) by

$$
\begin{aligned}
& (1-\alpha)[P(c)-P(-c)]-h P^{\prime}(c)+\frac{h^{2}}{2} P^{\prime \prime}(c) \\
& \quad=A|c|^{K} \int_{|c|}^{\infty}\left[P\left(c^{\prime}\right)+P\left(-c^{\prime}\right)\right] d c^{\prime}-[P(c)+P(-c)] A \frac{|c|^{K+1}}{K+1}
\end{aligned}
$$

This approximate integral equation is the one that we shall consider in the following. If we define $Q(c)$ and $R(c)$ by

$$
\begin{aligned}
& Q(c)=P(c)+P(-c) \\
& R(c)=P(c)-P(-c)
\end{aligned}
$$

then (21) can be decomposed into

$$
\begin{gathered}
-\frac{h}{2} R^{\prime}(c)+\frac{h^{2}}{4} Q^{\prime \prime}(c)=A\left[|c|^{K} \int_{|c|}^{\infty} Q\left(c^{\prime}\right) d c^{\prime}-\frac{|c|^{K+1}}{K+1} Q(c)\right] \\
2(1-\alpha) R(c)-h Q^{\prime}(c)+\frac{h^{2}}{2} R^{\prime \prime}(c)=0
\end{gathered}
$$

In fact using (20) and (22), one sees that in (25) the term which contains $R^{\prime \prime}$ is small compared with $R(c)$ and therefore (25) can be simplified into

$$
2(1-\alpha) R(c)-h Q^{\prime}(c)=0
$$

Then since $R(c)$ can be calculated in terms of $Q(c),(24)$ can be transformed into an equation for $Q(c)$ :

$$
-\frac{\alpha}{1-\alpha} \frac{h^{2}}{4} Q^{\prime \prime}(c)=A\left[|c|^{K} \int_{|c|}^{\infty} Q\left(c^{\prime}\right) d c^{\prime}-\frac{|c|^{K+1}}{K+1} Q(c)\right]
$$

Since $Q(c)$ is even, it is sufficient to study this equation for $c>0$. One can now transform this integral equation (27) into a differential equation. If we define $H(c)$ by

$$
H(c)=\int_{c}^{\infty} Q\left(c^{\prime}\right) d c^{\prime}
$$

Then (27) becomes

$$
\frac{\alpha}{1-\alpha} \frac{h^{2}}{4} H^{\prime \prime \prime}=A\left[c^{K} H+\frac{c^{K+1}}{K+1} H^{\prime}\right]
$$


Because $Q(c)$ is a probability distribution, $H(c), H^{\prime}(c), H^{\prime \prime}(c)$ have to vanish when $c \rightarrow \infty$. This allows us to integrate (29) and find that

$$
\frac{\alpha}{1-\alpha} \frac{h^{2}}{4} H^{\prime \prime}=A \frac{c^{K+1}}{K+1} H
$$

This means that if one knowns the solution $\psi(x)$ which decreases at $x \rightarrow \infty$ of the following equation:

$$
\frac{d^{2} \psi}{d x^{2}}=x^{K+1} \psi(x)
$$

Then the function $H(c)$ will be

$$
H(c)=\psi(\lambda c) / \psi(0)
$$

where $\lambda$ is given by

$$
\dot{\lambda}=\left(\frac{4 A}{K+1} \frac{1-\alpha}{\alpha} \frac{1}{h^{2}}\right)^{1 /(K+3)}
$$

and the $\psi(0)$ in the denominator of (32) is just there to normalize $H$.

So we see that if we can solve (31), then since $H(c)$ has the form (32), then $Q(c)$ will have the form (20) that we had assumed. Therefore, the assumption (20) was correct since one can find a $P(c)$ which solves the integral equation. We shall see in the Appendix what can be said about Eq. (31).

Let us now see what must be known about the function $\psi(x)$ in order to calculate the ground state energy. The ground state energy given in (17) can be transformed into

$$
\begin{aligned}
E= & \int_{-\infty}^{+\infty} J \rho(J) d J+\int_{-\infty}^{+\infty} P(c) d c\left[\int_{-\infty}^{c}(c-J) \rho(J) d J\right. \\
& \left.-\int_{-\infty}^{-c}(c+J) \rho(J) d J\right]
\end{aligned}
$$

which can be rewritten as

$$
\begin{aligned}
E= & \int_{-\infty}^{+\infty}|J| \rho(J) d J+\int_{-\infty}^{+\infty} P(c) d c\left[\int_{0}^{c}(c-J) \rho(J) d J\right. \\
& \left.-\int_{0}^{-c}(c+J) \rho(J) d J\right]
\end{aligned}
$$


This expression is completely exact. Now, using the fact that $P(c)$ is concentrated in a narrow region around 0 and that $\rho(J)$ can be replaced in this region by (3), one can write for small $h$

$$
E \simeq \int_{-\infty}^{+\infty}|J| \rho(J) d J+\frac{2 A}{(K+1)(K+2)} \int_{0}^{\infty} c^{K+2} Q(c) d c
$$

After an integration by parts, one gets

$$
E=\int_{-\infty}^{+\infty}|J| \rho(J) d J+\frac{2 A}{K+1} \int_{0}^{\infty} c^{K+1} H(c) d c
$$

Using now the form (32) of $H$,one sees that

$$
E=\int_{-\infty}^{+\infty}|J| \rho(J) d J+\frac{2 A}{K+1} \lambda^{-(K+2)} \mu_{K}
$$

where $\mu_{K}$ is a number defined by

$$
\mu_{K}=\frac{\int_{0}^{\infty} x^{K+1} \psi(x) d x}{\psi(0)}
$$

In the Appendix we shall show how one can calculate $\mu_{K}$. The result will be that

$$
\mu_{K}=(K+3)^{(K+1) /(K+3)} \frac{\Gamma((K+2) /(K+3))}{\Gamma(1 /(K+3))}
$$

Therefore using (33), (38), and (40), one gets

$$
\begin{aligned}
E= & \int|J| \rho(J) d J+2\left[\frac{A}{K+1}\right]^{1 /(K+3)}\left(\frac{\alpha}{1-\alpha} \frac{h^{2}}{4}\right)^{(K+2) /(K+3)} \\
& \times(K+3)^{(K+1) /(K+3)} \frac{\Gamma((K+2) /(K+3))}{\Gamma(1 /(K+3))}
\end{aligned}
$$

Then the magnetization can be obtained from

$$
m=\frac{\partial E}{\partial h}
$$

and one finds formula (5). 


\section{CONCLUSION}

It is interesting to compare the prediction of formula (5) with other known results. First if one takes blindly the limit $K \rightarrow \infty$, one finds that

$$
m \simeq h \frac{\alpha}{1-\alpha}
$$

This result was already found in the $\pm J$ case. ${ }^{(6)}$ It should probably hold for any distribution of $J_{i j}$ whose support does not contain 0 .

Second we can compare our result (5) with the numerical calculation of Chen and $\mathrm{Ma},{ }^{(13)}$ who determined the constant $C$ numerically (One should be careful in comparing their result with ours since they have a factor 2 in their Hamiltonian). If one takes their best fit with our definition of $h$, they claim that

$$
m \simeq 0.74(A h)^{1 / 3}
$$

whereas our formula (5) gives

$$
m \sim \frac{2^{2 / 3}}{3^{1 / 6}} \frac{[\Gamma(2 / 3)]^{2}}{\pi}(A h)^{1 / 2} \sim 0.7714887 \cdots(A h)^{1 / 3}
$$

Since they have only considered 12 samples of $10^{4}$ spins, one can say that the agreement is completely satisfactory.

We have also done a numerical calculation for a chain of $10^{6}$ spins with $\rho\left(J_{i j}\right)=1 / 2$ for $\left|J_{i j}\right|<1$ and we found

$$
\begin{array}{ll}
\text { for } h=10^{-4} & m / h^{1 / 3}=0.760 \pm 0.017 \\
\text { for } h=4 \times 10^{-4} & m / h^{1 / 3}=0.792 \pm 0.013
\end{array}
$$

So again the agreement seems rather good.

Haim Sompolinsky informed us that by an approach similar to the one described in the present paper, he was able to generalize our results to the low-temperature case. ${ }^{(14)}$

Lastly, it is interesting to notice that the fact that the exponents depend on the shape of the distribution $\rho\left(J_{i j}\right)$ has also been observed numerically in higher dimension. ${ }^{(15)}$

\section{ACKNOWLEDGMENTS}

We would like to thank D. Mukamel and H. Sompolinsky for discussions and encouragement which motivated this work. One of us (B.D.) 
would like to thank the Department of Physics of the Weizmann Institute where this work started. One of us (E.G.) is grateful to the Royal Society for financial support.

\section{APPENDIX}

In this Appendix, we study the solution of the differential equation (31) and we calculate the constant $\mu_{K}$ defined by (39).

The differential equation

$$
\frac{d^{2} \psi}{d x^{2}}=x^{K+1} \psi
$$

has in general two independent solutions. Because $\psi$ has to decrease for $x \rightarrow \infty$, we have to choose the one which decreases.

It is possible to express this solution in terms of Bessel functions:

$$
\psi(x)=\sqrt{x} K_{1 /(K+3)}\left(\frac{2}{K+3} x^{(K+3) / 2}\right)
$$

where the function $K_{v}$ is a Bessel function which can be written as

$$
K_{v}(z)=\sqrt{\pi} \frac{[(1 / 2) z]^{v}}{\Gamma(v+1 / 2)} \int_{1}^{\infty} e^{-z t}\left(t^{2}-1\right)^{v-1 / 2} d t
$$

However one does not need to be an expert in Bessel functions to obtain the expression of $\mu_{k}$.

Let us define $I_{\alpha}$ by

$$
I_{\alpha}=\int_{0}^{\infty} x^{\alpha} \psi(x) d x
$$

Then using the differential equation, one finds that

$$
\alpha(\alpha-1) I_{\alpha-2}=I_{\alpha+K+1}
$$

The general solution of this equation has the following form:

$$
I_{\alpha}=(K+3)^{2 \alpha /(K+3)} \Gamma\left(\frac{\alpha+2}{K+3}\right) \Gamma\left(\frac{\alpha+1}{K+3}\right) G\left(\frac{\alpha}{K+3}\right)
$$

where we have just used the fact that $\Gamma(z+1)=z \Gamma(z) . G(x)$ is a priori an arbitrary periodic function of period 1 . In the limit $x \rightarrow \infty$, one can solve equation (A1) easily:

$$
\psi(x) \sim x^{-[(K+1) / 4]} \exp \left[-\frac{2}{K+3} x^{(K+3) / 2}\right]
$$


For large $\alpha, I_{\alpha}$ can be estimated by a saddle point method

$$
I_{\alpha} \simeq \int_{0}^{\infty} x^{\alpha-(K+1) / 4} \exp \left[-\frac{2}{K+3} x^{(K+3) / 2}\right] d x
$$

and then one finds that the function $G$ is a constant. Therefore

$$
I_{\alpha}=G(K+3)^{2 \alpha /(K+3)} \Gamma\left(\frac{\alpha+2}{K+3}\right) \Gamma\left(\frac{\alpha+1}{K+3}\right)
$$

Once one knows the $I_{\alpha}$, it is easy to find the constant $\mu_{K}$ using the fact that when $\alpha \rightarrow-1$,

$$
I_{\alpha} \sim \frac{\psi(0)}{1+\alpha} \sim(K+3)^{-2 /(K+3)} \Gamma\left(\frac{1}{K+3}\right) \frac{K+3}{1+\alpha} G
$$

The result is of course independent of $G$

$$
\mu_{K}=(K+3)^{(K+1) /(K+3)} \frac{\Gamma((K+1) /(K+3))}{\Gamma(1 /(K+3))}
$$

\section{REFERENCES}

1. W. Kinzel and K. Binder, Phys. Rev. Lett. 50:1509 (1983); Phys. Rev. B 29:1300 (1984); J.

D. Reger, K. Binder, and W. Kinzel, Phys. Rev. B 30:4028 (1984).

2. K. Binder and A. P. Young, Phys. Rev. B 29:2684 (1983).

3. A. J. Bray and M. A. Moore, J. Phys. C 17:L463 (1984); J. Phys. C 17:613 (1984).

4. A. Vilenkin, Phys. Rev. B 18:1474 (1978).

5. V. Brandt and W. Gross, Z. Phys. B31:237 (1978).

6. B. Derrida, J. Vannimenus, and Y. Pomeau, J. Phys. C11:4749 (1978).

7. M. Puma and J. F. Fernandez, Phys. Rev. B 18:1391 (1978).

8. I. Morgenstern, K. Binder, and A. Baumgartner, J. Chem. Phys. 69:253 (1978).

9. G. Grinstein and D. Mukamel, Phys. Rev. B 27:4503 (1983); R. A. Pelcovits and D. Mukamel, Phys. Rev. B 28:5374 (1983).

10. R. Bruinsma and G. Aeppli, Phys. Rev. Lett. 50:1494 (1983); G. Aeppli and R. Bruinsma, Phys. Lett. 97A:117 (1983).

11. B. Derrida and H. J. Hilhorst, J. Phys. A 16:2641 (1983).

12. C. de Calan, J. M. Luck, T. M. Nieuwenhuizen, and D. Petritis, J. Phys. A 18:501 (1985).

13. H. H. Chen and S. K. Ma, J. Stat. Phys. 29:717 (1982).

14. H. Sompolinsky, private communication.

15. Ho-Fai Cheung and W. L. Mc Millan, J. Phys. C16:7027 (1983); J. Phys. C16:7033 (1983). 